\title{
Paper
}

\section{Histopathological study of Dengue Haemorrhagic Fever}

\author{
$K$ A P Idirisinghe
}

\begin{abstract}
Introduction: Dengue Haemorrhagic Fever (DHF) is a severe febrile disease caused by a Flavivirus.Four serotypes of dengue virus (DEN 1-4) are present and the large majority of DHF occurs following a secondary infection with a different serotype. About 0.5 million people in the world develop DHF annually with 24,000 deaths. Pathogenesis of the disease is still not fully understood. Target cells include dendritic reticulum cells, monocytes, lymphocytes, hepatocytes, and vascular endothelial cells.
\end{abstract}

Objectives: To describe the histopathological changes in the liver, lungs, heart, brain, spleen, gastrointestinal tract and kidneys in fatal DHF.

Materials and methods: Tissue from seventeen autopsies following DHF were processed routinely and stained with haematoxylin and eosin. Histopathological changes in the liver, lungs, heart, brain, spleen, gastrointestinal tract and kidneys were studied.

Results: Fifteen of 17 cases showed a liver pathology which included submassive necrosis (1/15), bridging necrosis (2/15), midzonal (3/15) and centrilobular necrosis (4/15), focal necrosis $(2 / 15)$, apoptosis (1/15) and macrovesicular steatosis (7/15). Pulmonary haemorrhage was present in 13 cases and pulmonary oedema in 6 cases. Glomerular congestion and haemorrhage were seen in 6 and medullary congestion with haemorrhage was present in 9 cases. Gastrointestinal mucosal haemorrhages were seen in 8 . One patient showed myocarditis and one showed myocardial haemorrhages. Cerebral oedema was present in 8 cases In 13 out of 17 cases the spleen showed red pulp congestion and haemorrhage.

Conclusion: DHF is a systemic viral infection causing multiorgan pathology. Liver, lungs and spleen are the most commonly affected organs.

(Key words: dengue haemorrhagic fever, autopsy, histopathology)

Department of Pathology, Teaching Hospital, Anuradhapura, Sri Lanka

Correspondence: KAP Idirisinghe e mail: pushpakodi@yahoo.com 


\section{Introduction}

Dengue fever is an arthropod borne viral infection caused by four antigenically distinct single stranded RNA viruses (DEN 1-4). The virus belongs to the family of flaviviridae and the genus flavivirus. The infection is transmitted through the bites of the mosquito Aedes aegypti and Aedes albopictus.

The first record of a disease resembling dengue fever was reported in $992 \mathrm{AD}$ in a Chinese medical encylopaedia (1). Unplanned urbanization in tropical developing countries, modern transportation, lack of effective mosquito control and globalization has facilitated the transmission of disease.Today, dengue is the most frequent arbovirus infection resulting inabout 50-100 million cases of dengue fever, 500,000 cases of Dengue Hemorrhagic Fever (DHF) and 24,000 deaths annually, worldwide (2). More than 100 countries are affected by dengue fever and an estimated 2.5 billion of people are at risk of infection (2).

The first serologically confirmed case of dengue in Sri Lanka was reported in 1962. In 2009, the country experienced the biggest dengue outbreak in its history. The magnitude of the outbreak was reflected by 35,007 suspected cases and 346 reported deaths. The national incidence rate of reported dengue cases for 2009 was 169 cases per 100,000 population (3).
There are only a few pathological studies on Dengue haemorrhagic fever and most include only a few cases. In Sri Lanka, such studies have not been undertaken. Therefore the aim of this study was to describe the histopathological changes in different organs in DHF which may possibly help clinicians in the understanding of the pathogenesis and management of this disease.

\section{Materials and methods}

Tissues obtained from seventeen autopsies of DHF patients were studied. Of these, six cases were PCR positive, 3 showed Dengue IgM positivity and 8 were clinically diagnosed as DHF. Tissue obtained at autopsy was fixed in 10\% formalin, processed routinely and stained with haematoxylin and eosin. Histopathological changes of the liver, lungs, heart, brain, spleen, gastrointestinal tract and kidneys identified under light microscopy were recorded.

\section{Results}

The ages of the patients in this study ranged from 7 months to 64 years. Ten patients were below 12 years of age. At autopsy pleural and peritoneal effusions were seen in eight cases. Eight had gastrointestinal haemorrhages. Congestion of the liver, lungs and spleen was seen macroscopically in 11,10 and 11 cases respectively. Cerebral oedema was present in 4 cases. 
On microscopic examination, histological Table 1. The liver was the most commonly changes were seen in the liver, lungs, spleen, affected organ (Figures 1-5). Fifteen of 17 cases kidney and heart. These have been outlined in showed a liver pathology (Table 2).

\section{Table 1. Histological findings in DHF}

\begin{tabular}{|l|c|}
\hline Organ and histological findings & No. of patients $\mathbf{N}=\mathbf{1 7})$ \\
\hline Lung & $13(77 \%)$ \\
Pulmonary haemorrhage & $6(35 \%)$ \\
Pulmonary oedema & \\
\hline Spleen & $13(77 \%)$ \\
Red pulp congestion and haemorrhage & $6(35 \%)$ \\
\hline Kidney & $9(53 \%)$ \\
Glomerular congestion and haemorrhage & \\
Medullary congestion and haemorrhage & $8(47 \%)$ \\
\hline Gastrointestinal tract & $1(6 \%)$ \\
Mucosal haemorrhage & $1(6 \%)$ \\
\hline Heart & $8(47 \%)$ \\
Myocarditis & \\
Myocardial haemorrhage & \\
\hline Brain & \\
Cerebral oedema & \\
\hline
\end{tabular}

Table 2. Histological changes in the liver

\begin{tabular}{|l|l|}
\hline Histological feature & Number of patients $(\mathbf{N}=\mathbf{1 5})$ \\
\hline Submassive necrosis & 1 \\
\hline Bridging necrosis & 2 \\
\hline Centrilobular necrosis & 4 \\
\hline Midzonal necrosis & 3 \\
\hline Focal necrosis & 2 \\
\hline Apoptosis & 1 \\
\hline Macrovesicularsteatosis & 7 \\
\hline Portal tract lymphocytes infiltrate & 15 \\
\hline
\end{tabular}




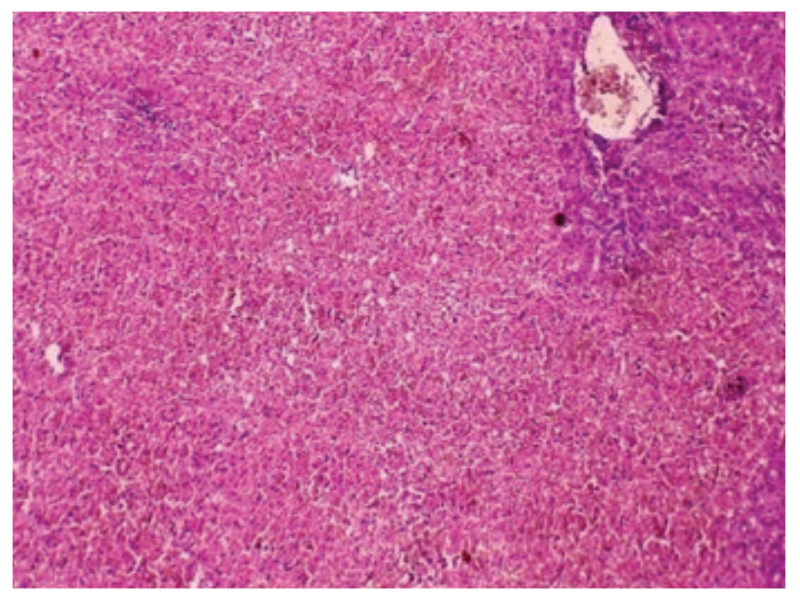

Fig.1. Liver- Submassive necrosis. (H\&Ex 100) 42 years. Dengue PCR positive

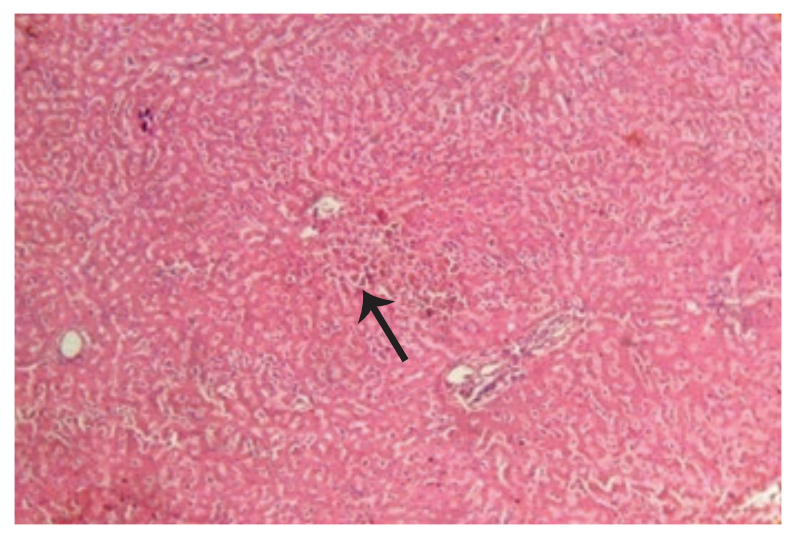

Fig.3. Liver: Focal necrosis(arrow). (H \& E x100) Clinically diagnosed as DHF.

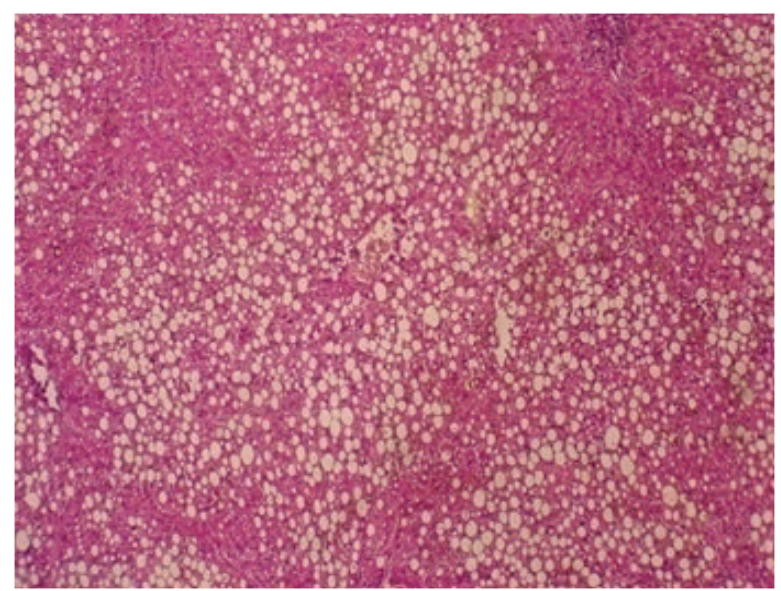

Fig. 5. Liver: Severe macrovesicular steatosis, (H \& E x 100) 14 years. Dengue PCR positive.

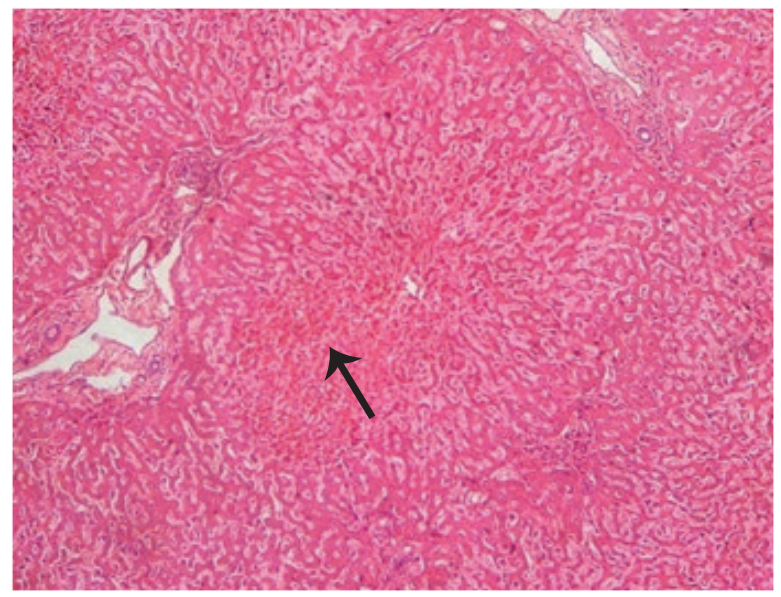

Fig. 2. Liver-Centrilobular necrosis (arrow) (H\&Ex100) 7 years. Dengue IgM positive.

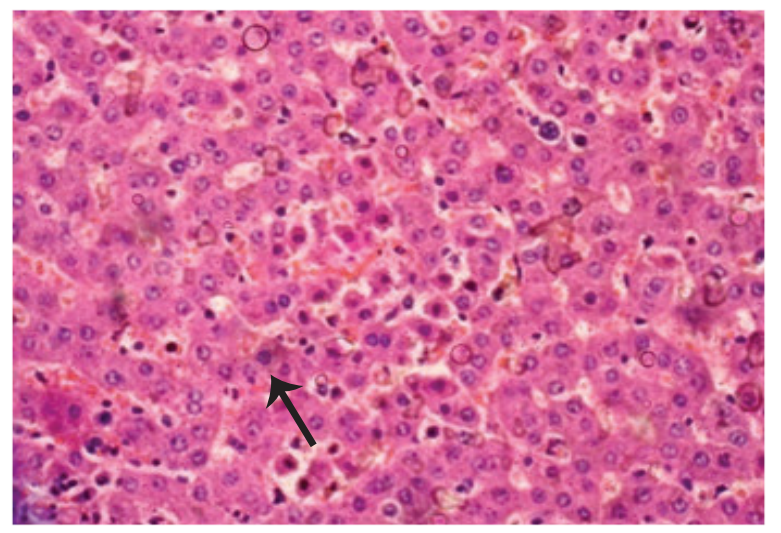

Fig. 4. Liver: Many apoptotic bodies. (H \& E $\mathrm{x} 400) 8$ years. Clinically diagnosed as DHF

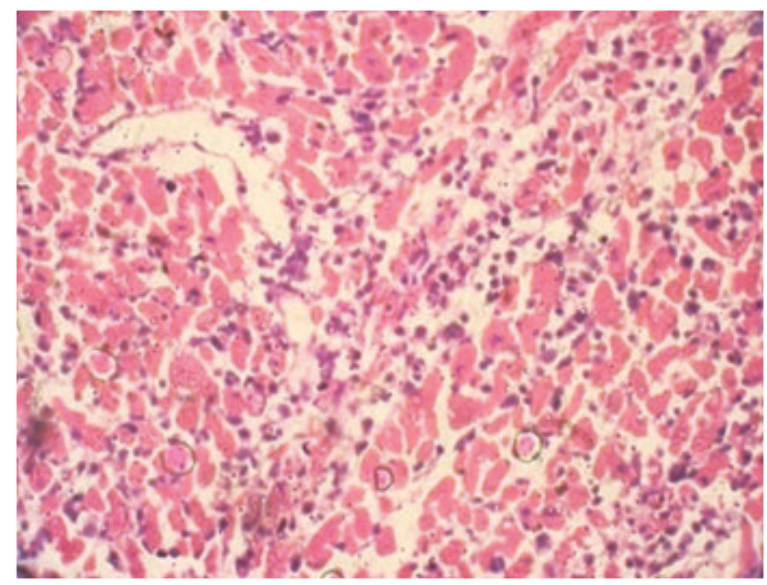

Fig. 6. Heart: Myocarditis. (H \& E x100). 19 years. Clinically diagnosed as DHF. 


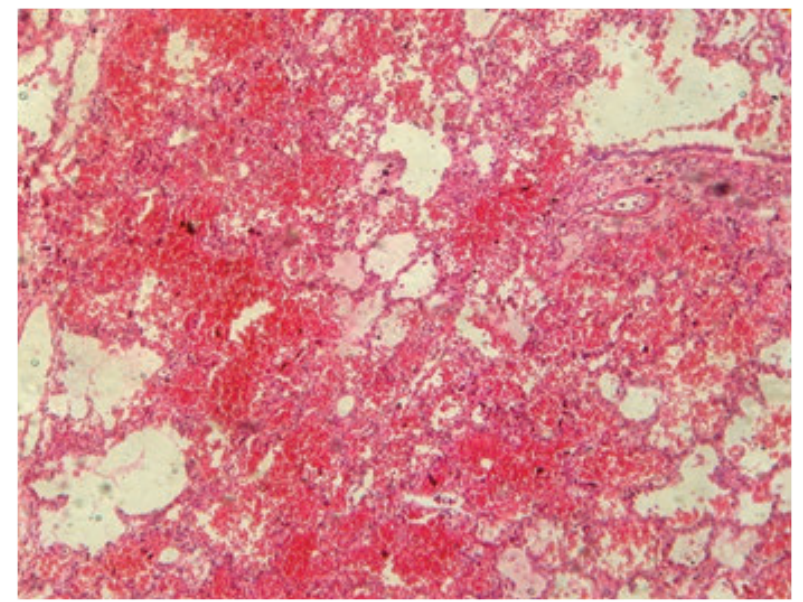

Fig. 7. Lungs: Pulmonary haemorrhages (H \& Ex100) 7 years. Dengue IgM positive

\section{Discussion}

Dengue fever is the most important arthropod born viral infection affecting more than 50-100 million people worldwide, annually (2). The infection is caused by four antigenically distinct single stranded RNA viruses (DEN 1-4) and infection with any serotype of the virus confers lifelong immunity to that particular serotype. The serotype cross reactive immunity is only transient and the host is susceptible to infection with the other three serotypes for the rest of his / her life.

Pathogenesis of dengue haemorrhagic fever is not fully understood due to the absence of a suitable animal host for experiments. The disease is thought to be caused by two immune mechanisms, production of non-neutralising antibodies that cross-react between different

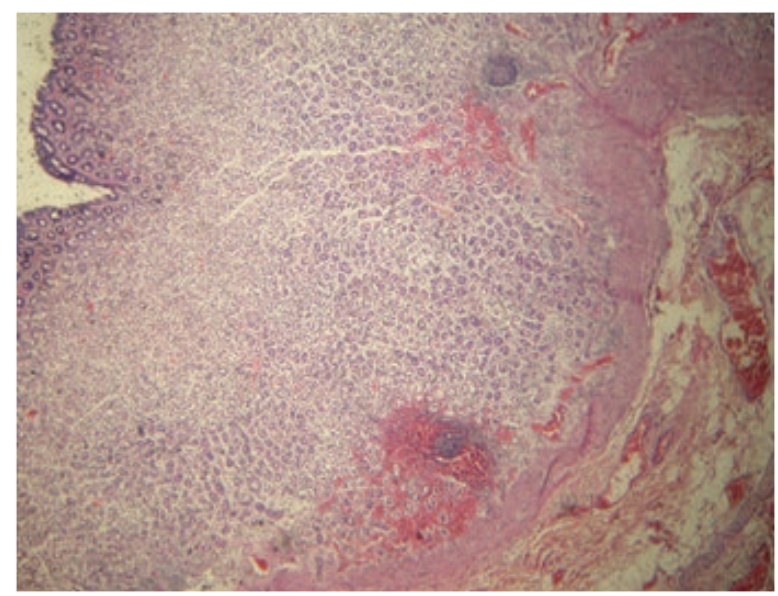

Fig. 8.GIT: Gastric mucosal haemorrhages $(\mathrm{H}$ \& Ex100) 12 years. Clinically diagnosed as DHF

serotypes of virus and activation of memory $\mathrm{T}$ cells sensitized in a previous infection. The target cells are predominantly cells of the reticulo endothelial system including spleen, liver, bone marrow, monocytes, lymphocytes, Kupffer cells and alveolar macrophages (4).

The clinical picture of dengue depends on both host and viral factors. A widespectrum of clinical manifestations ranging from a mild non-specific or a subclinical febrile illness to DHF and dengue shock syndrome (DSS) are seen. Atypical manifestations such as hepatitis, encephalitis, myocarditis, Reye's syndrome, haemolytic uraemic syndrome and thrombocytopenic purpura are also reported with dengue fever (5). DHF and DSS are the most severe manifestations of the infection and are associated with a poorer outcome and a mortality of about 
$5 \%$ (6). DHF is the cause for about 24,000 deaths

in subtropical and tropical countries, annually (2).

Liver is the most commonly affected organ in DHF and liver injury in DHF has been described since 1970 (7). Rathi et al studied 6 autopsies of DHF in India and all cases showed derangement in liver function tests indicating $100 \%$ liver involvement in their study (8). Raised levels of aspartate aminotransferase (AST) and alanine aminotransferase (ALT) have been observed in $98 \%$ and $37 \%$ of dengue patients, respectively (9). In the current study too, the liver was the most commonly affected organ and $88 \%(15 / 17)$ showed a liver pathology.

The liver is considered to be a direct target organ in dengue infection and viral particles have been demonstrated in hepatocytes. Histological changes reported in the liver in dengue fever include microvesicular steatosis and hepatocellular necrosis. A wide range of liver pathology was present in these cases and included submassive necrosis, bridging necrosis, centrilobular necrosis, midzonal necrosis, focal necrosis and apoptosis (Figures 1-5). Steatosis was not a significant finding in our study. All fifteen cases showed mild lymphocytic infiltrates of the portal tracts with no evidence of active hepatitis.
Acute liver failure is a severe life threatening condition in dengue infection, predisposing to haemorrhage, disseminated intravascular coagulation and encephalopathy $(10,11)$. Three patients in this study showed severe liver injury (submassive and bridging necrosis). However liver failure was not documented in these patient's records and this could be due to under diagnosis of liver failure in DHF. Also most cases with significant liver pathology (massive necrosis, bridging necrosis, zonal necrosis) had haemorrhagic manifestations in other organs. Seven of 8 cases with gastrointestinal bleeding, nine of thirteen cases with lung haemorrhages and eight of thirteen cases with splenic haemorrhages also showed significant liver necrosis. Therefore attention should be given to liver involvement in the management of dengue fever especially DHF.

Myocarditis is also a well recognized life threatening manifestation of dengue fever even though it is uncommon. In an autopsy study done in Sri Lanka five cases of myocarditis were reported in cytogenetically proven dengue fever (12). The histological changes they observed were heart muscle necrosis with inflammation and interstitial oedema of the myocardium (12). Also, in another study it has been shown that skeletal muscles and heart muscles are target 
cells in dengue infection (13). In this study, only one patient showed myocarditis (Figure 6) although several cases were clinically suspected as having myocarditis. One case showed myocardial haemorrhage.

In the lungs, alveolar congestion, septal haemorrhages, acute diffuse alveolar haemorrhages and pleural effusions have been reported with this infection $(12,14,15,16)$. Pulmonary haemorrhages were seen in thirteen cases in this study (Figure 7) and six showed pulmonary oedema. Nine of pulmonary haemorrhagic cases showed liver necrosis as well. However two cases with diffuse alveolar haemorrhages showed no significant liver pathology. Patients with DHF can present as acute respiratory distress syndrome and especially in dengue epidemics patients with acute respiratory distress syndrome should be investigated for dengue fever.

Spleen was another commonly affected organ and thirteen cases showed red pulp congestion and haemorrhage. Studies have shown depletion of cells in the periarterial lymphatic sheaths and the paracortical areas of the lymph nodes (17). Examination of the brain revealed cerebral oedema in eight cases but encephalitis was not seen in any of our cases.
Encephalitis though rare has been reported (18, 19). Eight cases showed gastrointestinal mucosal haemorrhages (Figure 8).

Serotyping of the virus in these cases was not done due to limited resources. Most of these cases were in 2009 (15/17) and the strain may have been more hepatotropic as fifteen out of seventeen autopsies showed a significant liver pathology. However, most of these cases showed multiorgan pathology. Liver (88\%), lungs $(82 \%)$ and spleen $(77 \%)$ were the commonly affected organs. Twelve cases showed involvement of more than three organs. The lungs and spleen were affected simultaneously in eight cases.

Therefore, dengue haemorrhagic fever should be regarded as a multiorgan infection and all systems should be considered in the management. Autopsy studies in every suspected dengue fever death should be performed and pathological findings should be documented along with the strain of the virus. These types of study will help to identify the pathology associated with the different serotypes of dengue virus and will greatly help in the management of dengue fever especially dengue haemorrhagic fever. 


\section{References}

1. Gubler DJ. Dengue and dengue hemorrhagic fever: Clinical Microbiology Review. 1998 July; 11 (3): $480-496$

2. WHO. Dengue and dengue haemorrhagic fever. Fact sheetNo 117, revised May 2008. Geneva, WorldHealth Organization, 2008 (http://www.who.int/ mediacentre/factsheets /fs117/en/).

3. Weekly epidemiological report, A publication of the Epidemiology Unit, Ministry of Healthcare and Nutrition, Sri Lanka. January 2010.Vol. 37, No.01: 1

4. Jessie K, Fong MY, Devi S, Lam SK, Wong KT. Localisation of dengue virus in naturally infected human tissues, by immunohistochemistry and in situ hybridisation. Journal of Infectious Diseases. 2004; 189:1411-1418.

5. Gulati S, Maheshwari A. Atypical manifestations of dengue. Tropical Medicine and International Health. 2007;12 (9):1087-1095.

6. Wilder-Smith A, Schwartz E: Dengue in travellers. New England Journal of Medicine. 2005; 353: 924-932.

7. George R., Lum LCS. Clinical spectrum of dengue infection. In: Gubler D.J. and Kuno G. (eds). Dengue and dengue hemorrhagic fever. Washington: $C a b$ International, 1997.

8. Rathi KR , Arora MM, Sahai K, TripathiS,Singh SP, Raman DK. Autopsy findings in fatal dengue haemorrhagic fever- 06 Cases. Medical Journal of Armed forces India, 2012; 1-6.
9. Nugyen TL, Nguyen TH, Tien NT: The impact of dengue hemorrhagic fever on liver function. Research in virology. 1997; 148:273-277.

10. Huerre MR, Lan NT, Marianneau P, Hue NB, Khun H, Hung NT et al . Liver histopathology and biological correlates in five cases of fatal dengue fever in Vietnamese children (abstract).Virchows archives. 2001 Feb; 438 (2):107-15.

11.Vinodh BN, Bammigatti C, Kumar A, Mittal V. Dengue fever with acute liver failure. Journal of postgraduate medicine. 2005: 51(4)322-323.

12. Weerakoon GADK, Kularatne AMS, Edussuriya DH, Kodikara KAS, Gunatilake PGL, Pinto VG et al. Histopathological diagnosis of myocarditis in a dengue outbreak in Sri Lanka, 2009. BMC Research Notes 2011, 4:268

13.Salgado DM, Eltit JM, Mansfield K, Panqueba C, Castro D, Vega MR et al. Heart and skeletal muscle are targets of dengue virus infection. Paediatric Infectious Diseases Journal 2010 March; 29(3):238-42.

14. Wang CC, Liu SF, Liao SC, Lee IK, Liu JW, Lin AS et al. Acute Respiratory Failure in Adult Patients with Dengue Virus Infection. American Journal of Tropical Medcine Hygeine. 2007 July: 77 (1) 151-158.

15. Marchiori E, Ferreira NJL, Bittencourt CN, de Araujo Neto CA, Zanetti G, Mano CM et al.Pulmonary haemorrhage syndrome associated with dengue fever, High-resolution computed tomography findings: a case report. Orphanet Journal of Rare 
Diseases. 2009; 4:8

16.Robert F, Morgan D, McAllister JK, Agan D, Horvath BK, Zimmerman L et al. Pulmonary Hemorrhage Syndrome Associated with an Autochthonous Case of Dengue Hemorrhagic Fever, Case Report. Southern Medical Journal: 2004 July; 97(7) 688-691

17.Aung-Khin M, Ma-Ma K, Thant-Zin Changes in the tissues of the immune system in dengue haemorrhagic fever Journal of Tropical Medicine and Hygeine 1975 December;78(12):256-61.

18.Lum LC, Lam SK, Choy YS, George R, Harun F. Dengue encephalitis: a true entity? American Journal of Tropical Medicine and Hygeine.1999March; 54(3):256-9.

19. RMR Nogueira AMB Filippis, JMO Coelh, PC Sequeira, HG Schatzmayr1, FG Paiva et al. Dengue virus infection of the central nervous system (cns): a case report from brazil. Southeast Asian Journal of Trophical Medicine in Public Health 2002 March; 33(1) $68-71$ 